\title{
Real-Time Imaging of Amygdalar Network Dynamics In Vitro Reveals a Neurophysiological Link to Behavior in a Mouse Model of Extremes in Trait Anxiety
}

\author{
Charilaos Avrabos, ${ }^{1,2}$ Sergey V. Sotnikov, ${ }^{1,3,4}$ Julien Dine, ${ }^{1,2}$ Patrick 0. Markt, ${ }^{1,3}$ Florian Holsboer, ${ }^{1}$ Rainer Landgraf, $, 1,3$ \\ and Matthias Eder ${ }^{1,2}$ \\ ${ }^{1}$ Max Planck Institute of Psychiatry, 80804 Munich, Germany, ${ }^{2}$ Research Group Neuronal Network Dynamics, ${ }^{3}$ Research Group Behavioral \\ Neuroendocrinology, and ${ }^{4}$ Department of Normal Physiology, Sechenov First Moscow State Medical University, 119991 Moscow, Russia
}

In humans and numerous other mammalian species, individuals considerably vary in their level of trait anxiety. This well known phenomenon is closely related to the etiology of several psychiatric disorders, but its neurophysiological basis remains poorly understood. Here, we applied voltage-sensitive dye imaging to brain slices from animals of the high (HAB), normal (NAB), and low (LAB) trait anxiety mouse model and investigated whether evoked neuronal activity propagations from the lateral (LA) to the central (CeA) amygdala differ in their relative strength among $\mathrm{HAB}, \mathrm{NAB}$, and $\mathrm{LAB}$ mice. For this purpose, we divided a real-time measure of neuronal population activity in the CeA by a respective measure obtained for the LA. This calculation yielded the metric "CeA/LA activity." Our data clearly demonstrate a positive correlation between trait anxiety levels evaluated by the elevated plus-maze test and CeA/LA activity. Moreover, we found reduced CeA/LA activity in HAB mice, which responded with decreased anxiety levels to an environmental enrichment and, inversely, detected increased anxiety levels and CeA/LA activity in LAB mice that experienced chronic mild stress. We did not observe differences in the spread of neuronal activity in the motor and visual cortex among HAB, NAB, and LAB animals. Collectively, these findings provide evidence that, in mammals, interindividual variability in trait anxiety is causally linked to individual variations in the physiological constitution of the LA-to-CeA circuitry that give rise to a differential regulation of neuronal signal flow through this fundamental input- output network of the amygdala.

\section{Introduction}

Interindividual variability in trait anxiety (IVTA) is a well known biological phenomenon that leads to partly profound individual differences in behavioral performance. In humans, IVTA furthermore is closely related to the etiology of anxiety disorders, depression, and several types of substance dependence (Holsboer and Ströhle, 2005; Hariri, 2009; Sandi and Richter-Levin, 2009). Consequently, intensive research has been performed to elucidate the biological causes of IVTA in mammals (mIVTA). This work yielded important insights into the genetic, biochemical, molecular, and neuroanatomical basis of mIVTA (Krömer et al., 2005; Duvarci et al., 2009; Hariri, 2009; Kim and Whalen, 2009; Montag et al., 2012). Moreover, fMRI and c-Fos expression studies have linked mIVTA with individual variations in threat (stress)induced neuronal activation in certain cortical and subcortical

\footnotetext{
Received June 6, 2013; revised Sept. 2, 2013; accepted Sept. 6, 2013

Author contributions: R.L. and M.E. designed research; C.A., S.V.S., J.D., and P.O.M. performed research; F.H. contributed unpublished reagents/analytic tools; C.A., S.V.S., P.O.M., and M.E. analyzed data; S.V.S., P.O.M., and M.E. wrote the paper.

We thank Carsten T. Wotjak for valuable comments on the manuscript.

The authors declare no competing financial interests.

Correspondence should be sent to Dr. Matthias Eder, Max Planck Institute of Psychiatry, Kraepelinstrasse 2-10, 80804 Munich, Germany. E-mail: eder@mpipsykl.mpg.de.

DOI:10.1523/JNEUROSCI.2397-13.2013

Copyright $\odot 2013$ the authors $\quad 0270-6474 / 13 / 3316262-06 \$ 15.00 / 0$
}

brain regions (Salomé et al., 2004; Hariri, 2009; Muigg et al., 2009). However, at the level of millisecond-scale neuronal network dynamics, the neurophysiological underpinnings of mIVTA still remain elusive.

A brain structure that is critically involved in the control of anxiety- and fear-related behaviors in mammals is the amygdala (Davis, 1992; Herry et al., 2008). Accordingly, this region of the limbic system has been evidenced to play a significant role in mIVTA (Hariri, 2009; Muigg et al., 2009). The lateral nucleus of the amygdala (LA) receives sensory information from the thalamus and cortex, and thus acts as a highly important input subfield (LeDoux, 2000; Pare and Duvarci, 2012). The central nucleus of the amygdala (CeA) in turn represents the main output station, and mediates autonomic and behavioral responses associated with anxiety and fear (Tye et al., 2011; Pare and Duvarci, 2012). Through a variety of neuronal interconnections, the LA signals directly and indirectly via the basal amygdala to the CeA (Pare and Duvarci, 2012). Based on these facts, we hypothesized that individual variations in input-output characteristics of the LA-to-CeA circuitry might account in part for mIVTA. To potentially obtain evidence for this scenario, we applied voltagesensitive dye imaging (VSDI) to brain slices from animals of the well validated high-anxiety-related behavior (HAB), normal anxiety-related behavior (NAB), and low-anxiety-related behavior (LAB) trait anxiety mouse model (Krömer et al., 2005), and 
A
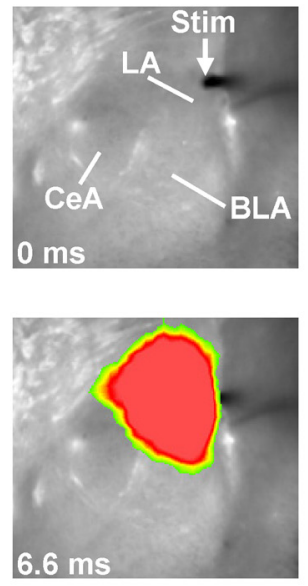

B

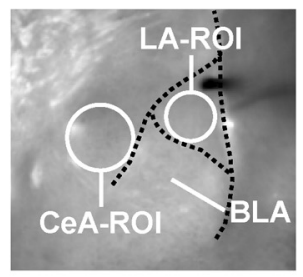

C

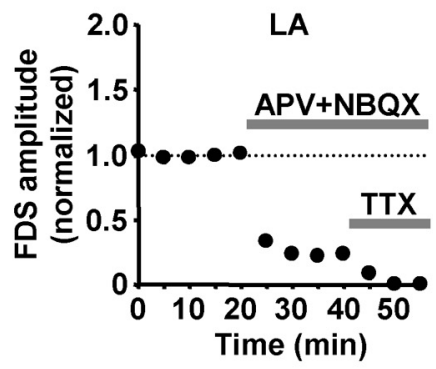

D

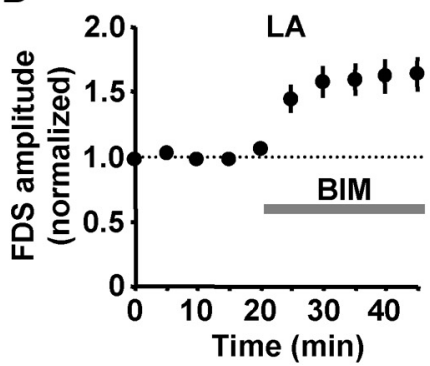

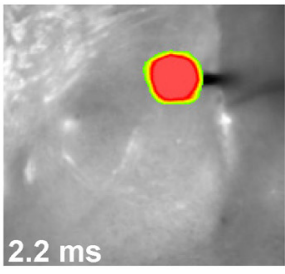
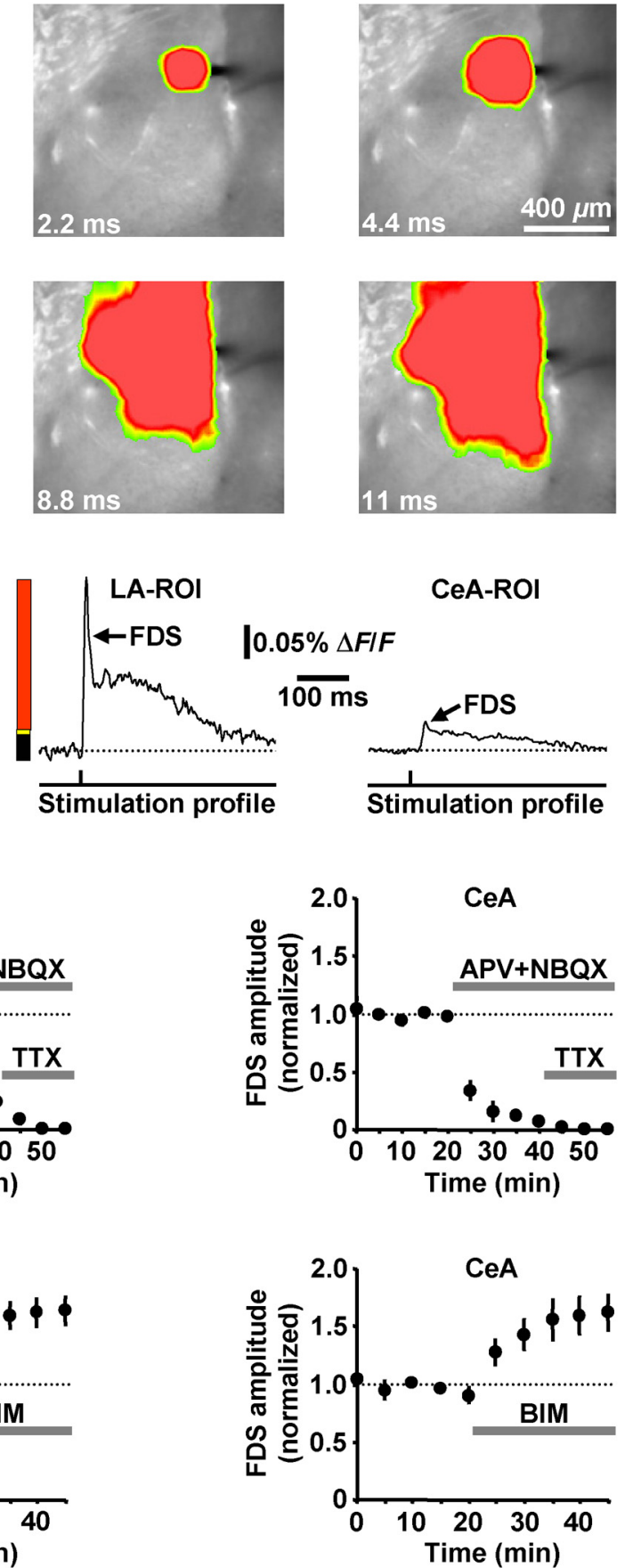

Figure 1. VSDI analysis and pharmacological validation of evoked neuronal activity propagations through the LA-to-CeA network. $\boldsymbol{A}$, VSDI filmstrip depicting the spread of neuronal activity triggered by an electrical stimulation pulse (Stim) delivered to the most dorsal part of the LA (for color bar, see $\boldsymbol{B}$ ). $\boldsymbol{B}$, ROI-based extraction of LA and CeA FDSs. $\boldsymbol{C}$, Coapplication of APV and NBQX to slices $(n=3)$ strongly diminished the LA and CeA FDSs. Residual FDS components were fully abolished by TTX. D, BIM enhanced the LA and CeA FDSs $(n=6)$. $A-D$, All experiments were performed in slices from NAB mice.

examined whether evoked neuronal activity propagations from the LA to the CeA differ in their relative strength among $\mathrm{HAB}$, $\mathrm{NAB}$, and $\mathrm{LAB}$ mice. In this study, we also included $\mathrm{HAB}$ and $\mathrm{LAB}$ animals that responded with decreased or increased anxiety levels to an environmental enrichment (EE) or chronic mild stress (CMS), respectively, thus covering both genetic predisposition to anxiety extremes and its environmental modification.

\section{Materials and Methods}

Animals. Experiments were approved by the Committee on Animal Health and Care of the local governmental body and performed in compliance with the guidelines for the care and use of laboratory animals set by the European Community. $\mathrm{HAB}$ and $\mathrm{LAB}$ mice, representing $\mathrm{CD}-1$ mice that have been selectively and bidirectionally bred for high- or low-anxiety-related behavior on the elevated plus-maze (EPM; >35 generations; Krömer et al., 2005), were obtained from the animal facilities of the Max Planck Institute of Psychiatry. "Normal" anxious CD-1 mice were purchased from Charles River and served as NAB animals (Krömer et al., 2005).

Behavioral testing. Animals were tested on postnatal day 49 (P49) on the EPM as described by Krömer et al. (2005). The percentage of time spent on the closed arms was used as a measure of the level of trait anxiety.

Environmental enrichment. EE comprised a combination of group housing (three mice per cage) and biologically relevant stimuli, including a $6 \pm 0.5$-cm-thick layer of wood chips, plastic inset $(22 \times 16 \times 8 \mathrm{~cm})$ and tunnel $(19.5 \times 6 \times 6 \mathrm{~cm})$, and wooden ladder and scaffold. These manipulations, combined with a bigger home cage $(55 \times 30 \times 20 \mathrm{~cm})$, allowed mice to accomplish highly motivated natural behaviors, while creating as few stressors as possible. To circumvent an increase in corticosterone/testosterone levels, accompanied by enhanced aggression, areas and toys that might lead to monopolization of cage structures and nutrition were omitted. Therefore, we did not supply the animals with a running wheel to bypass locomotor-induced anxiolysis. Furthermore, transfer of half of the nesting material during the weekly cage change allowed preservation of olfactory designation for the mice, which additionally ensured less aggression. EE comprised two $14 \mathrm{~d}$ periods: during P15-P28, pups and their respective dam were transferred for $6 \mathrm{~h} / \mathrm{d}$ to the EE, whereas on P29 pups were weaned, separated into groups of three, and transferred to the EE permanently until P42.

Chronic mild stress. CMS was applied during the same time period as EE and comprised a series of alternating stressors, including maternal separation (P15-P28), wet bedding, cage tilt, overcrowding, mild footshock, overnight illumination, white noise, and restraint stress (all during P29-P42). Food and water deprivation were excluded due to ethical reasons.

Preparation and staining of brain slices. Male 8- to 12-week-old mice were anesthetized with isoflurane and decapitated. All of the following steps were performed in ice-cold ACSF saturated with carbogen $\left(95 \% \mathrm{O}_{2} / 5 \% \mathrm{CO}_{2}\right)$. The ACSF consisted of the following (in mM): 125 $\mathrm{NaCl}, 2.5 \mathrm{KCl}, 25 \mathrm{NaHCO}_{3}, 1.25 \mathrm{NaH}_{2} \mathrm{PO}_{4}, 2$ $\mathrm{CaCl}_{2}, 1 \mathrm{MgCl}_{2}$, and 25 glucose. After decapitation, the brain was rapidly removed from the cranial cavity and $400-\mu \mathrm{m}$-thick coronal slices containing the amygdala or the motor or visual cortex were cut using a vibratome (HM650V; Thermo Scientific). Afterward, slices were incubated for $30 \mathrm{~min}$ at room temperature $\left(23-25^{\circ} \mathrm{C}\right)$. Subsequent staining of slices with the voltage-sensitive dye Di-4-ANEPPS was performed as described by Stepan et al. (2012). 
Brain slice experiments. In vitro experiments were performed at room temperature. Slices were continuously superfused with ACSF (4-5 $\mathrm{ml} / \mathrm{min}$ flow rate). Neuronal activity was evoked by square pulse electrical stimuli (200 $\mu$ s pulse width; $10-47.5 \mathrm{~V}$ ) delivered via a bipolar platinum/iridium cluster electrode (25 $\mu \mathrm{m}$ pole diameter; $\mathrm{FHC})$ to the most dorsal part of the LA (Fig. $1 A, B$ ) or layer $5\left(\mathrm{~L}_{5}\right)$ of the motor or visual cortex (see Fig. $4 A, B$ ). Drugs were administered by bath application.

VSDI. VSDI and data analysis were performed using the MiCAM02 hardware and software package (BrainVision). The microscope was equipped with the MiCAM02-HR camera and the $2 \times$ and $1 \times$ lens at the objective and condensing side, respectively. Acquisition settings were as follows: $88 \times 60$ pixel frame size, $36.4 \times 40.0 \mu \mathrm{m}$ pixel size, $2.2 \mathrm{~ms}$ sampling time, and $440 \mathrm{~ms}$ recording time [representing the time period during which the fractional change in fluorescence $(\Delta F / F)$ was calculated]. To reduce noise, seven acquisitions subsequently recorded at intervals of $10 \mathrm{~s}$ were averaged.

Processing and quantification of VSDI data. From recorded VSDI signals, $\Delta F / F$ was calculated. For all quantifications, $\Delta F / F$ values were spatially and temporally smoothed using a $3 \times$ $3 \times 3$ average filter. VSDI signals presented in images were smoothed by means of a $5 \times 5 \times 3$ average filter. Pixelation of images was reduced by the interpolation function of the MiCAM02 software. For analysis of neuronal population activity in amygdalar subregions, two standardized regions of interest (ROIs) were manually set according to anatomical landmarks. One circular ROI ( $r=4$ pixels) was positioned into the LA between the stimulation electrode and the basolateral nucleus (BLA). Another circular ROI ( $r=5$ pixels) covered the CeA (Fig. $1 B$ ). In the experiments performed in the motor and visual cortex, circular ROIs $(r=5$ or 3 pixels, respectively; 5 pixels in the motor cortex due to its larger size) were placed above the stimulation electrode into layers $2 / 3$ and 4 (see Fig. $4 B$ ). The average of smoothed $\Delta F / F$ values within a particular ROI served as the final measure of neuronal population activity.

Chemicals. Bicuculline methiodide (BIM), Di-4-ANEPPS, TTX, and all salts for the ACSF were purchased from Sigma-Aldrich. APV and NBQX were purchased from Ascent Scientific, and isoflurane from Abbott.

Statistics. Statistical analyses were run in SigmaStat, with significance declared at $p<0.05$ $\left.{ }^{*} p<0.05,{ }^{* *} p<0.01,{ }^{* * *} p<0.001\right)$. Data are given as the mean \pm SEM.

\section{Results}

First, we established an in vitro VSDI assay enabling the investigation of evoked neuronal activity propagations from the LA to the CeA. Pilot experiments revealed that such a spread of activity can be reliably triggered by electrical stimuli ( $\geq 10 \mathrm{~V}$ with the electrode used; $200 \mu$ s pulse width) delivered to the most dorsal part of the LA
A

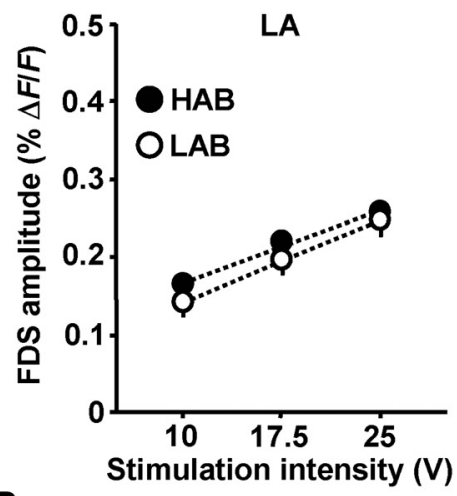

B

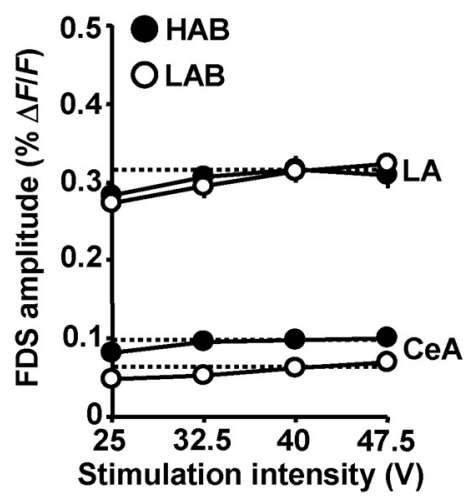

D
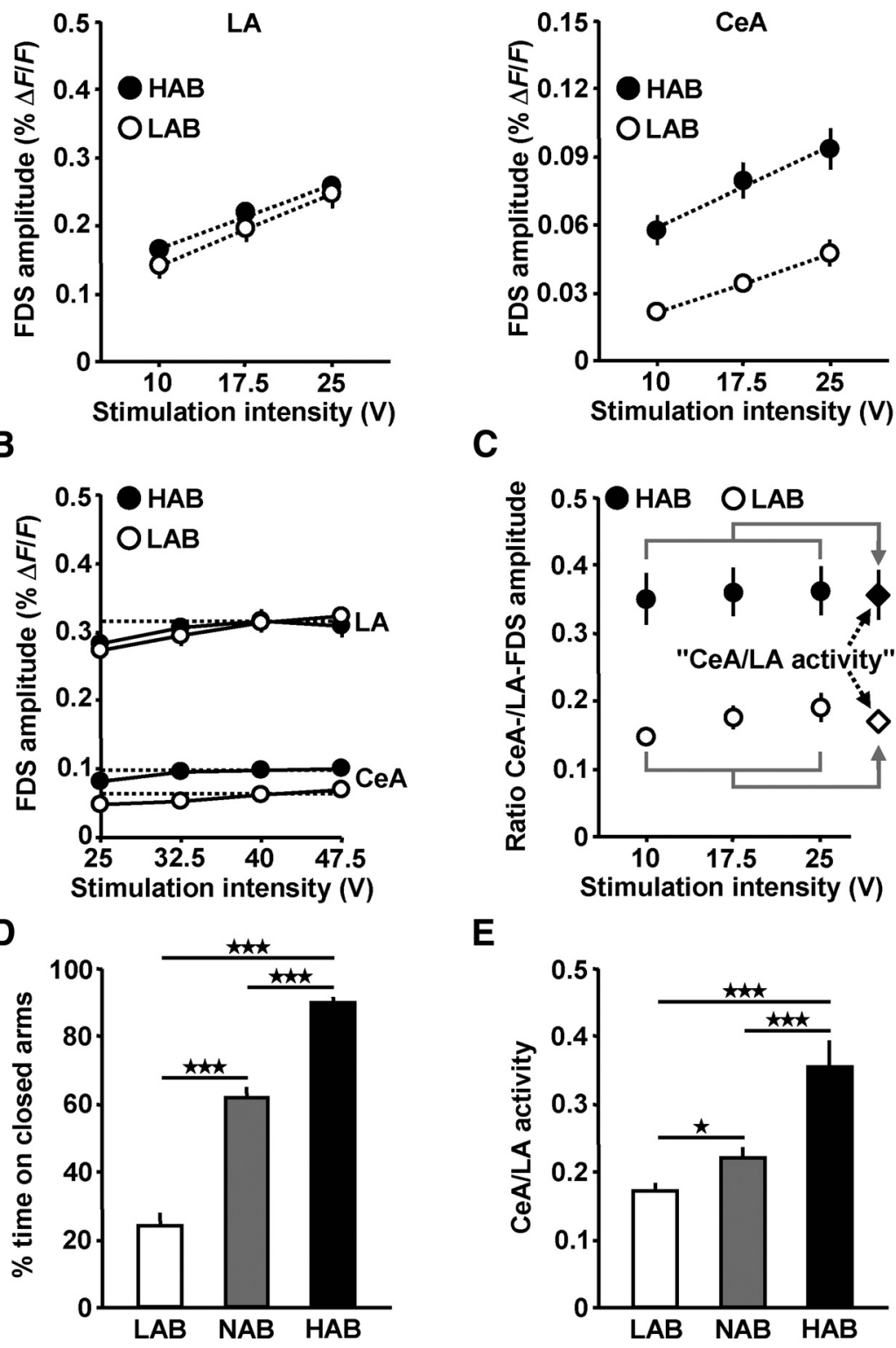

C

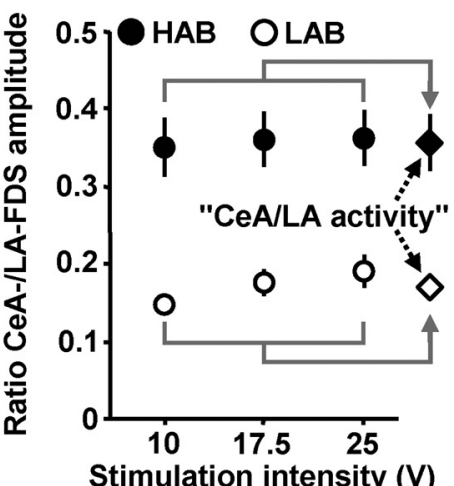

$\mathbf{E}$

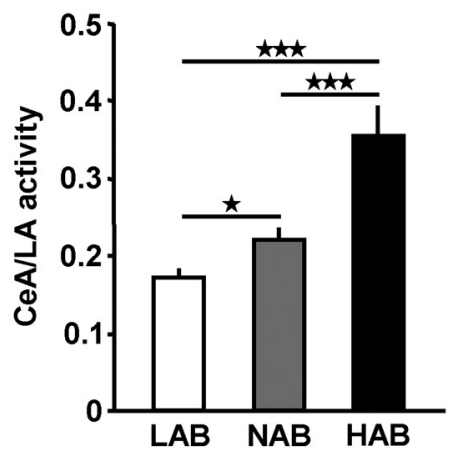

$\mathbf{F}$

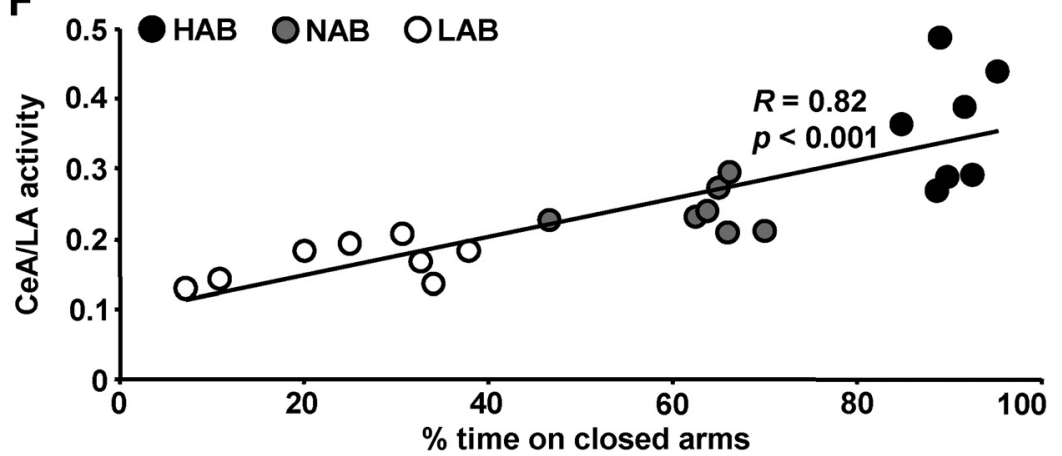

Figure 2. Evoked neuronal activity propagations through the $L A$-to-CeA network differ in their relative strength among $L A B$, $N A B$, and HAB mice. $A$, Quantification of peak amplitudes of the LA and CeA FDSs in slices from HAB and LAB mice (HAB: $n=12$ slices/7 animals; $L A B: n=12$ slices/8 animals). $B$, The LA and CeAFDSs became saturated with stimulation intensities $>25 \mathrm{~V}$ (HAB: $n=7$ slices/4 animals; LAB: $n=8$ slices/4 animals). Even with stimulation intensities $>25 \mathrm{~V}$, CeA activity was weaker in LAB mice. C, Calculation of the metric CeA/LA activity. D, Mean EPM anxiety levels of the $L A B, N A B$, and HAB mice tested (NAB: $n=7$ animals; for $H A B$ and $L A B$, see $\boldsymbol{A}$ ). $\boldsymbol{E}$, Quantification of $(e A / L A$ activity (NAB: $n=12$ slices/7 animals; for HAB and $L A B$, see $\boldsymbol{A}$ ). $\boldsymbol{F}$, Illustration of the positive correlation between the measure percentage of time on closed arms and CeA/LA activity (if two slices from an animal were analyzed, an average value of (eA/LA activity was calculated). 
A

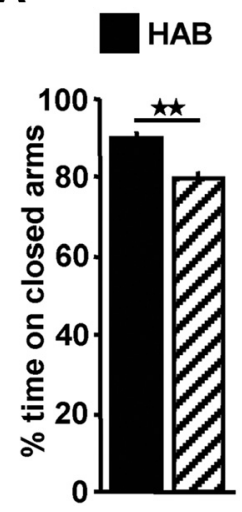

B
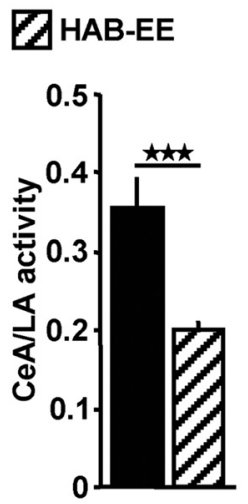

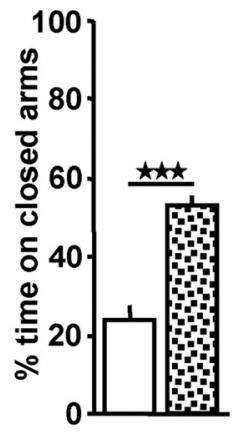

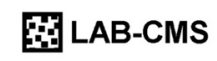

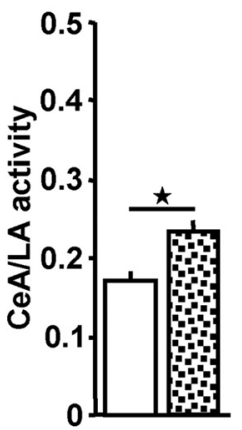

Figure 3. Effects of EE and CMS on EPM anxiety levels and CeA/LA activity in $H A B$ and $L A B$ animals. $A$, EE decreased EPM anxiety levels and CeA/LA activity in HAB mice (HAB: $n=7$ animals, 12 slices; HAB-EE: $n=7$ animals, 14 slices). $B$, CMS enhanced EPM anxiety levels and CeA/LA activity in LAB mice (LAB: $n=8$ animals, 12 slices; LAB-CMS: $n=7$ animals, 13 slices).

ments, we revealed that with higher stimulation intensities $(32.5,40$, and $47.5 \mathrm{~V}$ ) the LA and CeA FDSs become saturated (Fig. 2B; one-way repeated-measures ANOVAs: $F_{(3,18)}=8.9, p<0.001$ for HAB LA; $F_{(3,21)}=15, p<0.001$ for LAB LA; $F_{(3,18)}=12.1, p<0.001$ for $\mathrm{HAB} \mathrm{CeA}$; $F_{(3,21)}=12, p<0.001$ for LAB CeA; followed by Student-Newman-Keuls post hoc tests, 25 vs $40 \mathrm{~V}: p<0.001$ for HAB $\mathrm{LA} ; p<0.001$ for LAB LA; $p<0.001$ for $\mathrm{HAB}$ CeA; $p<0.01$ for LAB CeA; 40 vs $47.5 \mathrm{~V}: p>0.05$ for HAB LA; $p>0.05$ for $\mathrm{LAB} \mathrm{LA} ; p>0.05$ for HAB CeA; $p>0.05$ for LAB CeA). Even with stimulation intensities $>25 \mathrm{~V}$, CeA activity in slices from $\mathrm{LAB}$ animals was lower than in slices from $\mathrm{HAB}$ animals (Fig. $2 B ; 47.5 \mathrm{~V}: t_{(13)}=2.7$, $p<0.05$, two-tailed unpaired $t$ test). Since the ratios of the amplitude values did not

(Fig. 1A). This was, however, only the case for brain slices where on both sides the LA, the BLA, and the CeA were clearly visible. In most preparations, we obtained from each hemisphere one slice, which fulfilled this criterion. All in vitro experiments in the amygdala were conducted in these particular slices. As a measure of neuronal activity, we used ROI-extracted fast, depolarizationmediated imaging signals (FDSs; Fig. 1B). Stimulus-evoked FDSs in brain slice preparations have been shown to reflect neuronal action potentials as well as EPSPs (Tominaga et al., 2000; Airan et al., 2007; von Wolff et al., 2011; Stepan et al., 2012). Consistently, a pharmacological blockade of ionotropic glutamate receptors by APV $(50 \mu \mathrm{M})$ and NBQX $(5 \mu \mathrm{M})$ strongly diminished the LA and CeA FDSs (minutes $0-15$ vs $25-40: t_{(2)}=62.2, p<0.001$ for LA; $t_{(2)}=25.8, p<0.01$ for CeA; two-tailed paired $t$ tests). Residual FDS components were fully abolished by the voltage-gated $\mathrm{Na}^{+}$ channel antagonist TTX (1 $\mu \mathrm{M}$; Fig. $1 C)$. We further examined whether the stimulation paradigm used also entails an activation of inhibitory interneurons that confines the FDSs. For this purpose, we administered the $\mathrm{GABA}_{\mathrm{A}}$ receptor blocker BIM (10 $\left.\mu \mathrm{M}\right)$ to slices and found that this treatment indeed increases the LA and CeA FDSs (Fig. $1 D$; minutes $0-15$ vs $30-45: t_{(5)}=-5.1, p<$ 0.01 for LA; $t_{(5)}=-7.5, p<0.001$ for CeA; two-tailed paired $t$ tests]. These experiments, which were performed using a stimulation intensity of $17.5 \mathrm{~V}$ (for the rationale, see the next paragraph), additionally show that these FDSs remain constant over several consecutive evocations if no drug is added to the ACSF (Fig. 1C,D, baseline recordings).

Next, we investigated whether the activity propagations through the LA-to-CeA network differ in their relative strength among $\mathrm{LAB}, \mathrm{NAB}$, and $\mathrm{HAB}$ mice. We stimulated the LA at three intensities $(10,17.5$, and $25 \mathrm{~V}$; separated by 5 min time intervals) and divided the amplitude value of each CeA FDS by the amplitude value of the corresponding LA FDS. As exemplarily shown for the experiments conducted in HAB and LAB animals, both the LA and CeA FDSs linearly increased with increasing stimulation strength (Fig. 2A; one-way repeated-measures ANOVAs: $F_{(2,22)}=173.3, p<0.001$ for HAB LA; $F_{(2,22)}=54.7, p<0.001$ for LAB LA; $F_{(2,22)}=39.6, p<0.001$ for $\mathrm{HAB} \mathrm{CeA;} F_{(2,22)}=57.5$, $p<0.001$ for LAB CeA; followed by Student-Newman-Keuls post hoc tests, 10 vs $17.5 \mathrm{~V}: p<0.001$ for HAB LA; $p<0.001$ for LAB LA; $p<0.001$ for HAB CeA; $p<0.001$ for LAB CeA; 17.5 vs $25 \mathrm{~V}: p<0.001$ for HAB LA; $p<0.001$ for LAB LA; $p<0.01$ for $\mathrm{HAB} \mathrm{CeA} ; p<0.001$ for LAB CeA). In a separate set of experi- considerably vary among the $10,17.5$, and $25 \mathrm{~V}$ stimulations (Fig. $2 C$ ), we calculated the average ratios over all three stimulation intensities ("CeA/LA activity"; Fig. 2C, diamonds). Figure 2D shows the mean EPM anxiety levels of the LAB, NAB, and HAB mice tested, which typically markedly increased toward the HAB phenotype $\left(F_{(2,19)}=120, p<0.001\right.$, one-way ANOVA; followed by Student-Newman-Keuls post hoc tests, $p<0.001$ for all group comparisons). Intriguingly, a similar enhancement was observed for the metric CeA/LA activity (Fig. $2 E ; F_{(2,33)}=17.5, p<0.001$, one-way ANOVA; followed by Student-Newman-Keuls post hoc tests, $p<0.05$ for LAB vs NAB, $p<0.001$ for all other group comparisons). Accordingly, a linear regression analysis revealed a positive correlation between the behavioral measure percentage time on closed arms and CeA/LA activity (Fig. $2 F$ ).

It has been repeatedly demonstrated that anxiety in mice can be decreased or increased by an EE or prolonged stress exposure, respectively (Sztainberg et al., 2010; Hill et al., 2012). This is true even for genetically predisposed extremes in anxiety-related behavior, as HAB animals that grew up for 4 weeks (P15-P42) under EE conditions (HAB-EE) spent less time on the closed arms of the EPM, while LAB mice that were exposed to chronic mild stress during P15-P42 (LAB-CMS) displayed enhanced anxiety-related behavior (Fig. $3 A, B$, left panels). Note that the apparently moderate decrease in the percentage of time spent on closed arms (Fig. $3 A$ ) reflects an $\sim 100 \%$ increase in the measure percentage of time on open arms and, thus, represents a typical effect size (Sztainberg et al., 2010; HAB vs HAB-EE: $t_{(12)}=3.5, p<$ 0.01 ; LAB vs LAB-CMS: $t_{(13)}=6.2, p<0.001$; two-tailed unpaired $t$ tests). Home cage activity (monitored for $60 \mathrm{~h}$ ) did not differ among the HAB and HAB-EE groups as well as the LAB and LAB-CMS groups (data not shown), thus excluding the possibility that changes in basal locomotion accounted for the alterations in anxiety-related behavior. To potentially obtain evidence for a causal link between CeA/LA activity and EPM anxiety levels, we further examined whether the EE- and CMS-mediated behavioral changes are accompanied by parallel alterations in CeA/LA activity. Indeed, we detected lowered CeA/LA activity in slices from HAB-EE animals and observed the opposite scenario in LAB-CMS mice (Fig. $3 A, B$, right panels; $\mathrm{HAB}$ vs HAB-EE: $t_{(24)}=4.2, p<0.001$; LAB vs LAB-CMS: $t_{(23)}=2.3, p<0.05$; two-tailed unpaired $t$ tests).

Finally, we addressed the question of whether, in distributed networks usually not involved in anxiety behavior, the spread of 
neuronal activity also differs among $\mathrm{HAB}$, $\mathrm{NAB}$, and LAB mice. For this purpose, we stimulated layer 5 of the motor cortex (M2) and visual cortex (V1) at three intensities (10, 15, and $20 \mathrm{~V}$; Fig. 4A), extracted FDSs by ROIs covering layer $2 / 3$ $\left(\mathrm{L}_{2 / 3}\right)$ or $\mathrm{L}_{4}$ (Fig. $4 B$ ), and divided the amplitude value of each $\mathrm{L}_{2 / 3}$ FDS by the amplitude value of the corresponding $\mathrm{L}_{4}$ FDS. Both the $\mathrm{L}_{2 / 3}$ and $\mathrm{L}_{4}$ FDSs increased with increasing stimulation strength (data not shown). Since the ratios of the amplitude values did not considerably vary among the 10, 15 and $20 \mathrm{~V}$ stimulations (Fig. 4C), we calculated average ratios over all three stimulation intensities $\left(\mathrm{L}_{2 / 3} / \mathrm{L}_{4}\right.$ activity). Interestingly, $\mathrm{L}_{2 / 3} / \mathrm{L}_{4}$ activity was higher in V1 compared with M2, probably reflecting general differences in the physiological architecture between these networks. However, $\mathrm{L}_{2 / 3} / \mathrm{L}_{4}$ activity varied neither in $\mathrm{M} 2$ nor in $\mathrm{V} 1$ among $\mathrm{HAB}, \mathrm{NAB}$, and LAB mice (Fig. $4 D$; one-way ANOVAs: $F_{(2,20)}=0.2, p=0.82$ for $\mathrm{M} 2 ; F_{(2,20)}=0.46$, $p=0.64$ for $\mathrm{V} 1]$.

\section{Discussion}

In the present study, we addressed the question of whether mIVTA might partly result from individual differences in input-output characteristics of the LAto-CeA circuitry. For this purpose, we established a VSDI assay in mouse brain slices, allowing the investigation of several aspects of evoked neuronal activity propagations from the LA to the CeA. Using this assay in $\mathrm{HAB}, \mathrm{NAB}$, and $\mathrm{LAB}$ mice, we revealed a positive correlation between trait anxiety levels evaluated by the EPM test and CeA/LA activity (representing the metric that served as a quantifier of the relative strength of the activity propagations). Moreover, we detected reduced CeA/LA activity in HAB mice that exhibited decreased anxiety levels in response to an EE and, inversely, found enhanced anxiety levels and CeA/LA activity in LAB mice that experienced CMS. In addition, we did not observe differences in the spread of neuronal activity in $\mathrm{M} 2$ and $\mathrm{V} 1$ among $\mathrm{HAB}, \mathrm{NAB}$, and LAB animals. Together, these results provide evidence that mIVTA is causally linked to individual variations in the physiological constitution of the LA-to-CeA circuitry that give rise to a differential regulation of neuronal signal flow through this highly important input-output network of the amygdala.

Previous investigations performed in humans and mice have demonstrated by means of fMRI and c-Fos expression analysis that threat (stress)-induced neuronal activation in the amygdala positively correlates with the level of trait anxiety in an individual (Hariri, 2009; Muigg et al., 2009). However, the question of whether this connection relies on differences in the activity of brain structures that drive the amygdala and/or variations in the reactivity of amygdalar circuits to afferent inputs remains, to the
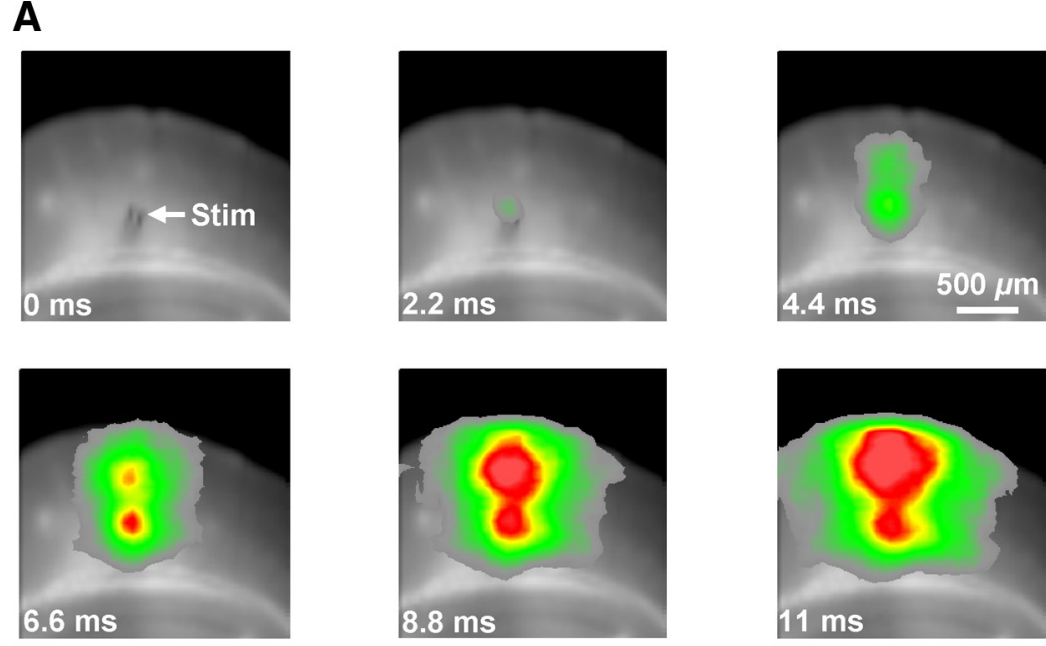

B
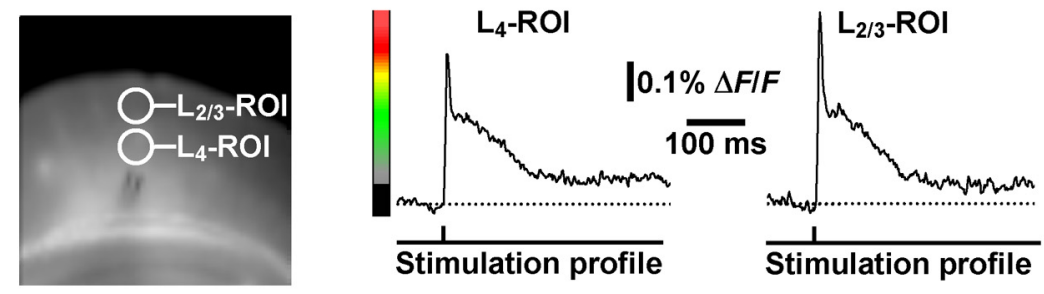

Stimulation profile

C

D

Figure 4. VSDI analysis of evoked neuronal activity propagations in the motor and visual cortex of HAB, NAB, and LAB mice. $\boldsymbol{A}$, VSDI filmstrip depicting the spread of neuronal activity triggered by an electrical stimulation pulse $(10 \mathrm{~V})$ delivered to $\mathrm{L}_{5}$ of $\mathrm{V} 1$ of a $\mathrm{LAB}$ animal (for color bar, see $\boldsymbol{B}$ ). $\boldsymbol{B}, \mathrm{R}$ Ol-based extraction of $\mathrm{L}_{4}$ and $\mathrm{L}_{2 / 3}$ FDSs. $\boldsymbol{C}, \boldsymbol{D}, \mathrm{L}_{2 / 3} / \mathrm{L}_{4}$ activity varied neither in $\mathrm{M} 2 \mathrm{nor}$ in $\mathrm{V} 1$ among $\mathrm{HAB}$, NAB, and LAB mice (M2: HAB, $n=8$ slices $/ 4$ animals; NAB, $n=7$ slices $/ 4$ animals; LAB, $n=8$ slices $/ 4$ animals; V1: $\mathrm{HAB}, n=7$ slices $/ 4$ animals; NAB, $n=8$ slices $/ 4$ animals; $\mathrm{LAB}, n=8$ slices $/ 4$ animals).

best of our knowledge, not conclusively answered until now. While our data cannot exclude the first scenario, they give support for an at least significant contribution of the second one.

Several previous studies (Airan et al., 2007; Refojo et al., 2011; Stepan et al., 2012) and the present work prove VSDI as a valuable tool for the exploration of millisecond-scale neuronal network dynamics and their alterations under pathology-like states, given that exaggerated anxiety, as seen in HAB mice (Krömer et al., 2005), represents one of the most frequent psychiatric conditions (Holsboer and Ströhle, 2005). VSDI therefore can help to attain the goal of relating neurophysiological processes at the molecular and cellular level to those exposed by brain imaging techniques (e.g., fMRI) and also behavioral phenotypes, both genetically and environmentally driven. Accordingly, a more intensive use of circuit-centered approaches is increasingly recommended 
(Karayiorgou et al., 2012). In this context, one can argue that our study most likely encountered a "circuit-level" mechanism contributing to the regulation of trait anxiety in mice and probably also other mammals. It is questionable whether this could have been achieved by classical microelectrode recordings since the imaging signals used in our analyses reflect neuronal activity scattered over areas of $\sim 10^{5} \mu \mathrm{m}^{2}$ (Fig. $1 B$ ).

Which variations in the physiological constitution of the LAto-CeA circuitry might account for the observed differences in CeA/LA activity? For instance, it has been shown that an increased inhibition of CeA neurons by medial paracapsular cells (resulting from an enhanced probability of transmitter release at glutamatergic synapses targeting these interneurons) decreases EPM anxiety levels in mice (Jüngling et al., 2008). Consistently, infusions of muscimol into the CeA were reported to exert an anxiolytic effect on the EPM in rats (Moreira et al., 2007). Both of these changes in GABA receptor-mediated inhibition most likely lead to a reduction of neuronal activity that is restricted to the CeA (Fig. 2, compare $A, C$ ). Thus, microstructural and/or functional variations in the amygdalar circuits that generate inhibitory inputs to the CeA could underlie the differences in the activity propagations investigated here. Furthermore, as evidenced by the combination of the findings from Sztainberg et al. (2010) and our observation that EE lowers CeA/LA activity, the strength of CRF receptor type 1 expression in the BLA might also play an important mechanistic role.

In conclusion, our data strongly suggest that mIVTA arises in part from individual variations in input-output characteristics of the LA-to-CeA circuitry. Hence, our study underscores this neuronal network as a promising target for novel therapeutic interventions for the treatment of pathological anxiety.

\section{References}

Airan RD, Meltzer LA, Roy M, Gong Y, Chen H, Deisseroth K (2007) Highspeed imaging reveals neurophysiological links to behavior in an animal model of depression. Science 317:819-823. CrossRef Medline

Davis M (1992) The role of the amygdala in fear and anxiety. Annu Rev Neurosci 15:353-375. CrossRef Medline

Duvarci S, Bauer EP, Paré D (2009) The bed nucleus of the stria terminalis mediates inter-individual variations in anxiety and fear. J Neurosci 29: 10357-10361. CrossRef Medline

Hariri AR (2009) The neurobiology of individual differences in complex behavioral traits. Annu Rev Neurosci 32:225-247. CrossRef Medline

Herry C, Ciocchi S, Senn V, Demmou L, Müller C, Lüthi A (2008) Switching on and off fear by distinct neuronal circuits. Nature 454:600-606. CrossRef Medline

Hill MN, Kumar SA, Filipski SB, Iverson M, Stuhr KL, Keith JM, Cravatt BF, Hillard CJ, Chattarji S, McEwen BS (2012) Disruption of fatty acid amide hydrolase activity prevents the effects of chronic stress on anxiety and amygdalar microstructure. Mol Psychiatry. Advance online publication. Retrieved September 9, 2013. doi:10.1038/mp.2012.90. CrossRef Medline

Holsboer F, Ströhle A (Eds) (2005) Anxiety and anxiolytic drugs. Berlin: Springer.

Jüngling K, Seidenbecher T, Sosulina L, Lesting J, Sangha S, Clark SD, Okamura N, Duangdao DM, Xu YL, Reinscheid RK, Pape HC (2008) Neuropeptide S-mediated control of fear expression and extinction: role of intercalated GABAergic neurons in the amygdala. Neuron 59:298-310. CrossRef Medline

Karayiorgou M, Flint J, Gogos JA, Malenka RC; Genetic and Neural Complexity in Psychiatry 2011 Working Group (2012) The best of times, the worst of times for psychiatric disease. Nat Neurosci 15:811-812. CrossRef Medline

Kim MJ, Whalen PJ (2009) The structural integrity of an amygdalaprefrontal pathway predicts trait anxiety. J Neurosci 29:11614-11618. CrossRef Medline

Krömer SA, Kessler MS, Milfay D, Birg IN, Bunck M, Czibere L, Panhuysen M, Pütz B, Deussing JM, Holsboer F, Landgraf R, Turck CW (2005) Identification of glyoxalase-I as a protein marker in a mouse model of extremes in trait anxiety. J Neurosci 25:4375-4384. CrossRef Medline

LeDoux JE (2000) Emotion circuits in the brain. Annu Rev Neurosci 23: 155-184. CrossRef Medline

Montag C, Reuter M, Weber B, Markett S, Schoene-Bake JC (2012) Individual differences in trait anxiety are associated with white matter tract integrity in the left temporal lobe in healthy males but not females. Neuroscience 217:77-83. CrossRef Medline

Moreira CM, Masson S, Carvalho MC, Brandão ML (2007) Exploratory behaviour of rats in the elevated plus-maze is differentially sensitive to inactivation of the basolateral and central amygdaloid nuclei. Brain Res Bull 71:466-474. CrossRef Medline

Muigg P, Scheiber S, Salchner P, Bunck M, Landgraf R, Singewald N (2009) Differential stress-induced neuronal activation patterns in mouse lines selectively bred for high, normal or low anxiety. PLoS One 4:e5346. CrossRef Medline

Pare D, Duvarci S (2012) Amygdala microcircuits mediating fear expression and extinction. Curr Opin Neurobiol 22:717-723. CrossRef Medline

Refojo D, Schweizer M, Kuehne C, Ehrenberg S, Thoeringer C, Vogl AM, Dedic N, Schumacher M, von Wolff G, Avrabos C, Touma C, Engblom D, Schütz G, Nave KA, Eder M, Wotjak CT, Sillaber I, Holsboer F, Wurst W, Deussing JM (2011) Glutamatergic and dopaminergic neurons mediate anxiogenic and anxiolytic effects of CRHR1. Science 333:1903-1907. CrossRef Medline

Salomé N, Salchner P, Viltart O, Sequeira H, Wigger A, Landgraf R, Singewald N (2004) Neurobiological correlates of high (HAB) versus low anxietyrelated behavior (LAB): differential Fos expression in HAB and LAB rats. Biol Psychiatry 55:715-723. CrossRef Medline

Sandi C, Richter-Levin G (2009) From high anxiety trait to depression: a neurocognitive hypothesis. Trends Neurosci 32:312-320. CrossRef Medline

Stepan J, Dine J, Fenzl T, Polta SA, von Wolff G, Wotjak CT, Eder M (2012) Entorhinal theta-frequency input to the dentate gyrus trisynaptically evokes hippocampal CA1 LTP. Front Neural Circuits 6:64. CrossRef Medline

Sztainberg Y, Kuperman Y, Tsoory M, Lebow M, Chen A (2010) The anxiolytic effect of environmental enrichment is mediated via amygdalar CRF receptor type 1. Mol Psychiatry 15:905-917. CrossRef Medline

Tominaga T, Tominaga Y, Yamada H, Matsumoto G, Ichikawa M (2000) Quantification of optical signals with electrophysiological signals in neural activities of Di-4-ANEPPS stained rat hippocampal slices. J Neurosci Methods 102:11-23. CrossRef Medline

Tye KM, Prakash R, Kim SY, Fenno LE, Grosenick L, Zarabi H, Thompson KR, Gradinaru V, Ramakrishnan C, Deisseroth K (2011) Amygdala circuitry mediating reversible and bidirectional control of anxiety. Nature 471:358-362. CrossRef Medline

von Wolff G, Avrabos C, Stepan J, Wurst W, Deussing JM, Holsboer F, Eder M (2011) Voltage-sensitive dye imaging demonstrates an enhancing effect of corticotropin-releasing hormone on neuronal activity propagation through the hippocampal formation. J Psychiatr Res 45:256-261. CrossRef Medline 